\title{
Aplicación de la metodología iRAP y el software ViDA-iRAP en un tramo de autopista en México
}

\section{Application of iRAP methodology and ViDA-iRAP software on a section of highway in Mexico}

\section{M. en I. Antonio Hurtado-Beltrán}

Universidad Michoacana de San Nicolás de Hidalgo-Facultad de Ingeniería Civil-Departamento de Vías Terrestres, México

ahurbel@gmail.com

Secretaría de Comunicaciones y Transportes-Dirección General de Servicios Técnicos-Unidad Regional de Servicios Técnicos

Morelia, México

ahurtadb@sct.gob.mx

\section{Ing. Margarita Serna-Rodríguez}

Secretaría de Comunicaciones y Transportes-Dirección General de Servicios Técnicos-Unidad Regional de Servicios Técnicos

Morelia, México

msernaro@sct.gob.mx

\section{M. en I. Julio Alejandro Chávez-Cárdenas}

Universidad Michoacana de San Nicolás de Hidalgo-Facultad de Ingeniería Civil-Departamento de Vías Terrestres, México

juliochavezc@hotmail.com

Fecha de recepción: 23 de setiembre de 2014 / Fecha de aprobación: 11 de noviembre de 2014

\section{RESUMEN}

Los traumatismos causados por los accidentes de tránsito en el mundo se han convertido en las últimas décadas en un problema creciente de salud pública a nivel mundial que demanda una atención inmediata. El programa iRAP (International Road Assessment Programme) plantea la posibilidad de realizar una evaluación de la seguridad que tienen los usuarios dentro de las redes viales con el objetivo de establecer medidas que reduzcan el gran número de muertos y heridos graves que actualmente se están presentando en los países en vías de desarrollo. En el presente trabajo se plasma la aplicación del programa iRAP y la herramienta informática de reciente desarrollo ViDA-iRAP, para definir las "contramedidas" de seguridad vial que habrán de implementarse en un camino de altas especificaciones de la red troncal de carreteras de México, considerando para el análisis y los resultados secciones de $100 \mathrm{~m}$ de longitud.

PALABRAS CLAVE: iRAP, ViDA-iRAP, contramedidas, seguridad vial, tránsito, vialidad, vehículo, usuario.

\section{ABSTRACT}

Injuries caused by traffic accidents in the world have become in recent decades a growing public health problem worldwide that demand immediate attention. The iRAP methodology (International Road Assessment Programme) presents the possibility of a safety assessment that the users have within the road networks with the goal to establish measures to reduce the large number of deaths and serious injuries that currently occurring in the developing countries. This paper shows the application of the program $i R A P$ and the newly developed software tool ViDA-iRAP, to define "countermeasures" of road safety to be implemented on a path of high specifications of the main road network in Mexico, considering for the analysis and results sections of $100 \mathrm{~m}$ in length.

KEYWORDS: iRAP, ViDA-iRAP, countermeasures, road safety, traffic, road, vehicle, user. 


\section{INTRODUCCIÓN}

Los accidentes de tránsito se han convertido, en las últimas décadas, en un problema creciente de salud pública a nivel mundial. Los traumatismos causados por el tránsito actualmente representan una de las tres causas principales de mortalidad en las personas de 5 a 44 años de edad, y es la primera causa de muerte entre jóvenes de 15 a 29 años de edad (OMS, 2009). Aunque las tasas de accidentes de tránsito en los países de ingresos altos se han estabilizado o han ido disminuyendo, los datos indican que en la mayor parte de los países la problemática mundial de traumatismos por accidentes de tránsito sigue aumentando.

Cada año en el mundo mueren 1.3 millones de personas como consecuencia de los accidentes en las vías de tránsito y 50 millones sufren algún tipo de traumatismo. La Organización Mundial de la Salud (OMS) estima que, a menos que se tomen medidas inmediatas, para el año 2030 el número de víctimas mortales en las vías de circulación se incrementará a 2.4 millones por año.

El 11 de mayo del 2011 la Asamblea General de la Organización de las Naciones Unidas, proclamó el periodo 2011-2020 como el "Decenio de Acción para la Seguridad Vial", con el objetivo de estabilizar y reducir las cifras previstas de víctimas mortales en accidentes de tránsito en todo el mundo. Gobiernos, organismos internacionales, organizaciones de la sociedad civil y empresas privadas de más de 100 países, incluido México, se comprometieron a llevar a cabo planes de acción concretos o de promulgar una nueva legislación en materia de seguridad vial.

Una parte importante de los esfuerzos generados para atender el problema de seguridad vial en el mundo recae en el International

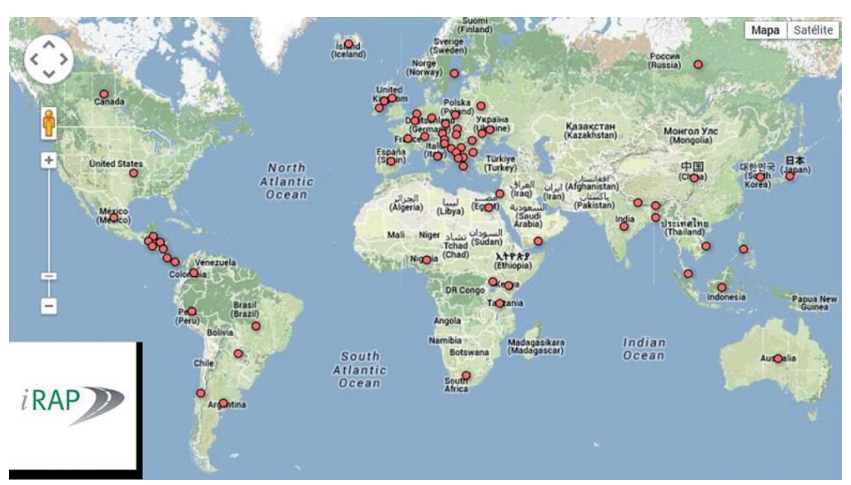

Figura 1. Presencia del programa iRAP en el escenario mundial, (cortesía de http://www.irap.org)
Road Assessment Programme (iRAP), traducido en español como Programa Internacional de Evaluación de Carreteras, se trata de una organización benéfica registrada en Inglaterra reconocida por el Grupo de Colaboración de las Naciones Unidas para la Seguridad Vial.

El programa iRAP fue formado en 2006 como una organización que aglutinó la experiencia y los resultados generados en programas de investigación tales como el EuroRAP (Europa, 1999), usRAP (USA, 2004) y AusRAP (Australia, 2006), (iRAP, 2013b). El objetivo primordial del programa iRAP es establecer medidas que mejoren la seguridad de todos los usuarios dentro de la infraestructura vial, enfocándose principalmente a los países de medios y bajos ingresos, puesto que en estos países se concentran más del $90 \%$ de las víctimas mortales de accidentes de tránsito en el mundo, aún cuando solo tienen el $48 \%$ del parque vehicular (OMS, 2009).

El programa iRAP se aplicó inicialmente (2006 y 2007) como un estudio piloto en Malasia, Chile, Costa Rica y Sudáfrica. Estos países ofrecían ejemplos de una gran variedad de escenarios relativos a la seguridad vial, desde la gran cantidad de motociclistas en Malasia, hasta carreteras de una sola calzada con altos límites de velocidad en Sudáfrica. Esto permitió desarrollar y poner a prueba las herramientas creadas en el programa, mejorando y ampliando su rango de aplicación bajo diferentes escenarios (iRAP, 2009a y 2009b). Desde entonces el programa iRAP ha evolucionado hasta su versión más reciente, “iRAP Versión 3", lanzada en el año 2012, y con la cual se han evaluado más de 150,000 km de carreteras en el mundo (iRAP, 2013b). En la Figura 1 se puede apreciar la presencia que tiene actualmente el programa iRAP en el escenario mundial.

Como resultado de la aplicación de este programa se puede obtener la información enlistada a continuación:

- Tablas y mapas con valoraciones por "estrellas" (calificación de tramos carreteros), que indican la seguridad de las carreteras para ocupantes de vehículos, motociclistas, ciclistas y peatones.

- Una base de datos con un inventario de carreteras con más de 30 atributos inspeccionados en la red de carreteras.

- Una estimación del número de personas que resultan muertas o gravemente heridas en cada una de las carreteras inspeccionadas.

- Un programa recomendado de contramedidas rentable para toda la red, para ser considerado por las autoridades locales y entidades a cargo de la financiación. 


\section{ESTRATEGIA NACIONAL DE SEGURIDAD VIAL EN MÉXICO}

Según datos de la OMS al 2010, cada año en México los accidentes de tránsito cobran la vida de más de 16,500 personas, y más de 167,000 sufren lesiones graves y/o discapacitantes. En México el costo anual derivado de los accidentes de tránsito asciende a más de $\$ 450,000$ millones de pesos, lo que representa el 3.6\% del Producto Interno Bruto del país (iRAP, 2013a).

A mediados del año 2011, la Secretaría de Comunicaciones y Transportes y la Secretaría de Salud suscribieron un acuerdo en el que se daba a conocer la "Estrategia Nacional de Seguridad Vial 2011-2020" que se aplicaría en México. Dicha estrategia tiene como objetivo general reducir un 50\% las muertes, así como reducir al máximo posible las lesiones y discapacidades generadas por accidentes de tránsito (DOF, 6 de junio de 2011). La estrategia considera los siguientes ejes de acción:

- Fortalecer la gestión de la seguridad vial mediante una serie de acciones multisectoriales en los diferentes órdenes de gobierno.

- Evaluar y mejorar la infraestructura vial y de transportes.

- Fomentar el uso de vehículos más seguros.

- Mejorar el comportamiento de los usuarios de las vías de tránsito.

- Promover la mejora de los servicios de atención médica derivados de accidentes de tránsito.

En lo referente a la evaluación y mejora de la infraestructura vial, la Secretaría de Comunicaciones y Transportes (SCT) signó un convenio con la organización iRAP para aplicar su programa y metodología en la evaluación de la red carretera del país (iRAP, 2012a). Esta dependencia, a través de su Dirección General de Servicios Técnicos, tutela y administra la aplicación del programa iRAP, con el cual, se han inspeccionado ya cerca de 45,000 km de vías troncales y 20,000 km de vías secundarias; utilizando la "Clasificación por Estrellas" para medir el riesgo de la infraestructura vial y tomar las medidas pertinentes a fin de reducirlo sistemáticamente. Junto con los datos de iRAP, las inspecciones están recolectando información acerca de los parámetros funcionales y estructurales del pavimento, a fin de utilizarlos para la gestión y planeación óptima de los activos y de las acciones de conservación.

El proyecto también será el primero en utilizar el software en línea "ViDA-iRAP", que ofrece velocidades de procesamiento significativamente mayores y capacidad de elaboración de reportes más exhaustivos (iRAP, Web-Ref. 9).
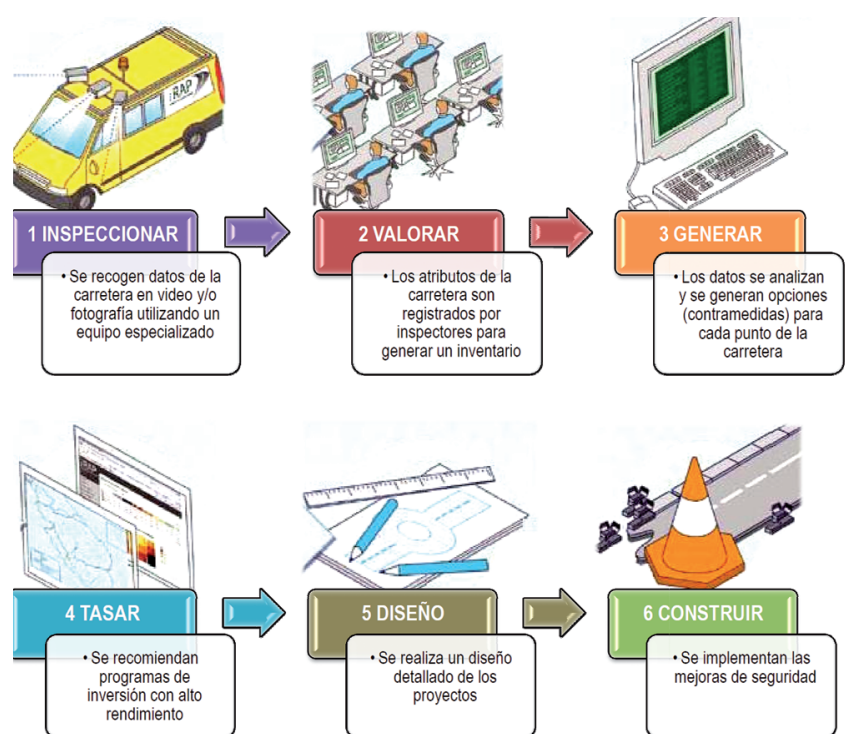

Figura 2. Etapas de la metodología iRAP, (modificado de iRAP, 2009a)

\section{METODOLOGÍA IRAP}

La idea medular de la metodología iRAP es la valoración o calificación por "estrellas" (SRS, Star Rating Score) para medir la seguridad vial de toda una red de carreteras o parte de ella. Esto proporciona una plataforma valiosa cuando no se dispone de registros sobre datos de accidentes, o cuando son inexactos o escasos. La metodología está conformada por las etapas mostradas en la Figura 2.

La aplicación de la metodología comienza con el levantamiento en video y/o fotografía de la mayor parte de los elementos de la infraestructura carretera, utilizando para ello equipo especializado que permita obtener un ángulo de visión mínimo de 150 grados y el posicionamiento GPS de cada imagen recabada. $\mathrm{Al}$ ir montado el equipo sobre un vehículo automotor que circula a velocidades de operación el levantamiento puede realizarse con relativa rapidez. Un equipo de inspectores o codificadores acreditados por iRAP dan lectura al levantamiento fotográfico para registrar en gabinete más de 30 diferentes atributos (características) observados en cada sección de la carretera, considerados como los más influyentes en la probabilidad de ocurrencia y la gravedad de accidentes de tránsito.

Los atributos de la carretera son puntuados y combinados con base a la severidad y al peligro utilizando una serie de factores y ecuaciones (para mayor detalle consultar las referencias iRAP, 2013d y 2013e) cuyos resultados reflejan la seguridad general que la carretera ofrece a ocupantes de vehículos, motociclistas, ciclistas o peatones. A las puntuaciones (SRS) obtenidas 
se les asignan estrellas, de 1 a 5, permitiendo con ello una representación cartográfica. Las carreteras más seguras (4 y 5 estrellas) tienen atributos de seguridad vial que son apropiados para las velocidades de tráfico prevalecientes. La infraestructura carretera en un camino seguro podría incluir la separación de sentidos de circulación con una mediana o una barrera, buena señalización en caminos e intersecciones, carriles anchos y acotamientos pavimentados, libre de peligros laterales no frangibles en caminos sin barreras de orilla, e infraestructura y espacios adecuados para ciclistas y peatones, tales como banquetas, ciclovías y cruces peatonales. Las carreteras menos seguras ( 1 y 2 estrellas) no tienen atributos de seguridad vial que sean apropiados para las velocidades de tráfico prevalecientes. Estas son a menudo las carreteras de una sola calzada con curvas frecuentes e intersecciones, carriles estrechos, acotamientos sin pavimentar, delineación deficiente, accesos irregulares y peligros laterales sin protección, tales como árboles, postes y terraplenes escarpados cercanos a la orilla de la carretera. Asimismo, no tienen infraestructura y espacios adecuados para los ciclistas y peatones.

Una vez asignada la calificación por "estrellas" a cada sección de la carretera, se detectan tramos o puntos críticos y se analizan para plantear alternativas (contramedidas) que incrementen la calificación por "estrellas" obtenida inicialmente, lo cual, se traduce en una mejora en términos de seguridad para los usuarios. Las contramedidas aplicables con la mayor relación beneficio-costo son seleccionadas para establecer un programa de inversión para la carretera analizada (o a nivel de la red carretera), y se realiza el diseño detallado del proyecto con la cuantificación y la ubicación puntual de las contramedidas seleccionadas. Finalmente, se materializan en el sitio las medidas necesarias para incrementar el nivel de seguridad de la carretera.

\section{SOFTWARE EN LÍNEA VIDA-iRAP}

El software en línea ViDA-iRAP (Versión Beta) es una herramienta informática con plataforma en la web recientemente desarrollada dentro del programa iRAP para agilizar y hacer más eficiente el procesamiento de tareas que habitualmente demandan una cantidad de tiempo considerable si se ejecutan manualmente (Ver Figura 3), como lo son la calificación por "estrellas" (medida de la seguridad), la generación de contramedidas (alternativas disponibles), y los análisis beneficio-costo (programa de inversión).

Debido principalmente al tamaño de su red carretera, México será el primer país en utilizar el software ViDA-iRAP (Versión Beta). El software utiliza la tecnología informática de última generación denominada "nube informática" para administrar todos los datos de la evaluación de la red carretera, y ofrece las herramientas y los servicios para el procesamiento de la información. Entre las principales características del software ViDA-iRAP (Versión Beta) están (iRAP, 2012b):

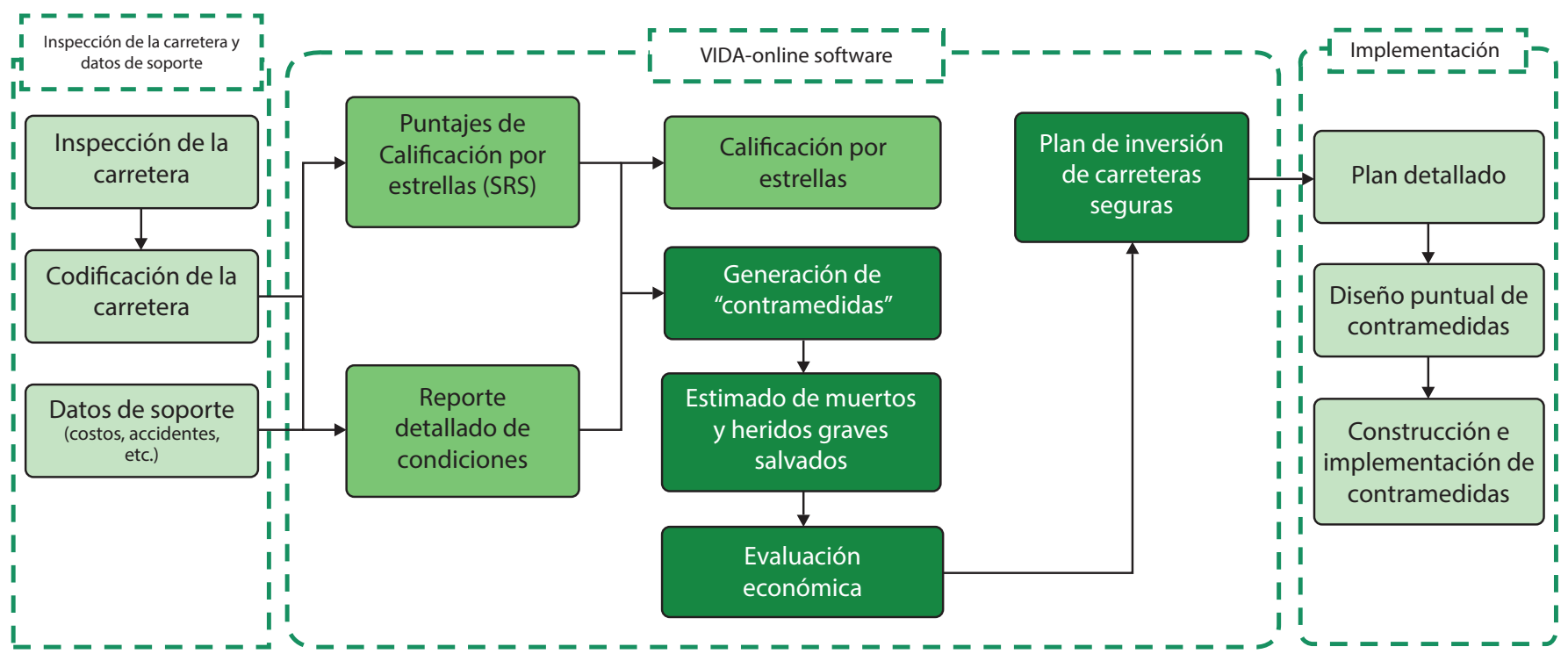

Figura 3. Participación del software ViDA-iRAP dentro del proceso de la metodología iRAP 
- Guía de análisis paso a paso para que los proveedores y el personal de gobierno puedan aplicar los protocolos.

- Precisión en la evaluación de datos.

- Mejoras del modelo y personalizaciones para adaptarse a los entornos locales.

- Análisis de pronóstico ante modificaciones en las condiciones existentes.

- Reportes personalizados y la disponibilidad de filtros flexibles de la información.

- Facilita el intercambio, colaboración y comunicación entre todos los miembros del proyecto.

\section{CASO DE APLICACIÓN}

\section{Tramo seleccionado}

Para obtener el riesgo que tiene el usuario en un camino de altas especificaciones y las contramedidas recomendadas para reducirlo, se seleccionó del Portal ViDA-iRAP el tramo de la autopista federal de cuota Maravatío-Zapotlanejo (Mex-15D) entre el km 202+000 y el km 240+000, considerando para el análisis únicamente el "sentido 1" (de Maravatío hacia Zapotlanejo) bajo la condición "Ocupante de Vehículo". Es preciso señalar que el sistema ViDA-iRAP permite hacer un análisis independiente para cada uno de los usuarios de la vía (ocupante de vehículo, motociclista, ciclista y peatón).

\section{Localización}

El tramo estudiado se localiza al Noreste de la ciudad de Morelia, entre las coordenadas geográficas $19^{\circ} 52^{\prime}$ y $19^{\circ} 54^{\prime}$ de latitud Norte, y $100^{\circ} 46^{\prime}$ y $101^{\circ} 09^{\prime}$ de longitud Oeste, cruza las carreteras Acámbaro-Zinapécuaro y Morelia-Salamanca. En la Figura 4 puede observarse la ubicación del tramo seleccionado.

\section{Características del tramo}

Las características físicas y geométricas del tramo seleccionado son las siguientes:

- Camino tipo ET-4.

- Velocidad de proyecto de $110 \mathrm{~km} / \mathrm{h}$.

- 4 carriles (dos carriles por sentido).

- Cuerpos separados.

- Ancho de carril de $3.65 \mathrm{~m}$.

- Ancho de acotamiento interior de $1.00 \mathrm{~m}$.

- Ancho de acotamiento exterior de $2.40 \mathrm{~m}$.

- Ancho de faja separadora central variable.

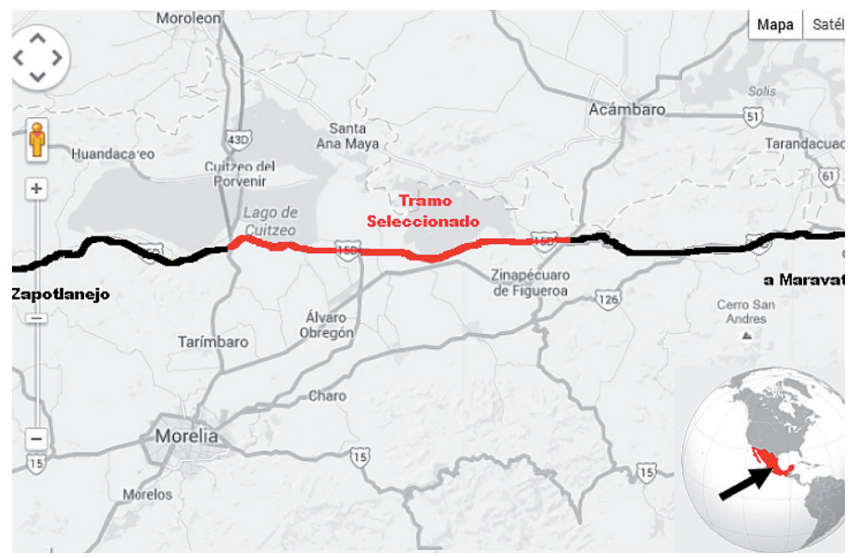

Figura 4. Localización del tramo seleccionado, (modificado de http://vida.irap.org)

\section{Topografía}

Del km 202+000 al km 206+000 es lomerío suave, del km 206+000 al km 207+000 es plano, del km 207+000 al km 209+000 es lomerío suave, del km 209+000 al km 213+000 es plano, del km 213+000 al km 217+700 es lomerío fuerte, del km 217+700 al km $228+500$ es plano, del km 228+500 al 240+000 es lomerío fuerte.

\section{INFORMACIÓN ARROJADA POR EL SISTEMA VIDA-iRAP}

A continuación se presenta la información proporcionada por el sistema ViDA-iRAP para el tramo seleccionado. La información recabada corresponde únicamente a la condición "Ocupante de Vehículo". La información proporcionada por el sistema está basada en los datos obtenidos de la inspección realizada en la autopista seleccionada en el año 2012, los cuales han sido cargados a la plataforma. Es importante señalar que los datos de la evaluación de la red troncal de carreteras se están actualizando anualmente, para llevar un control de los avances que se van consiguiendo en cuanto al mejoramiento de la seguridad vial.

\section{Mapas del sistema ViDA-iRAP}

El software ViDA-iRAP utiliza los datos de la evaluación cargados a la plataforma del sistema para definir la calificación por "estrellas" de la carretera. Para ello, el sistema calcula el puntaje "SRS" (Star Rating Scores) por segmentos de 100 m de longitud, tomando como referencia la condición de seguridad más crítica de cada segmento analizado. El puntaje "SRS" considera todos los factores de riesgo involucrados y la severidad del daño potencial para cada tipo de usuario. La seguridad del camino disminuye en la medida que se incrementa el puntaje "SRS". La calificación por "estrellas" y su relación con el puntaje "SRS" puede apreciarse en la Tabla 1 . Se considera que un camino con 
Tabla 1. Bandas "SRS" y colores de la calificación por "estrellas", (modificado de iRAP, 2013c)

\begin{tabular}{|c|c|c|c|}
\hline \multirow{2}{*}{$\begin{array}{c}\text { Calificación por } \\
\text { estrellas }\end{array}$} & $\begin{array}{c}|c| \\
\text { Pcupante Vehículo y } \\
\text { motociclista }\end{array}$ & Peatón & Ciclista \\
\hline 5 & 0 a 2.5 & 0 a 5 & 0 a 5 \\
4 & 2.5 a 5 & 5 a 15 & 5 a 10 \\
3 & 5.0 a 12.5 & 15 a 40 & 10 a 30 \\
\hline 2 & 12.5 a 22.5 & 40 a 100 & 30 a 60 \\
\hline 1 & $>22.5$ & $>100$ & $>60$ \\
\hline
\end{tabular}

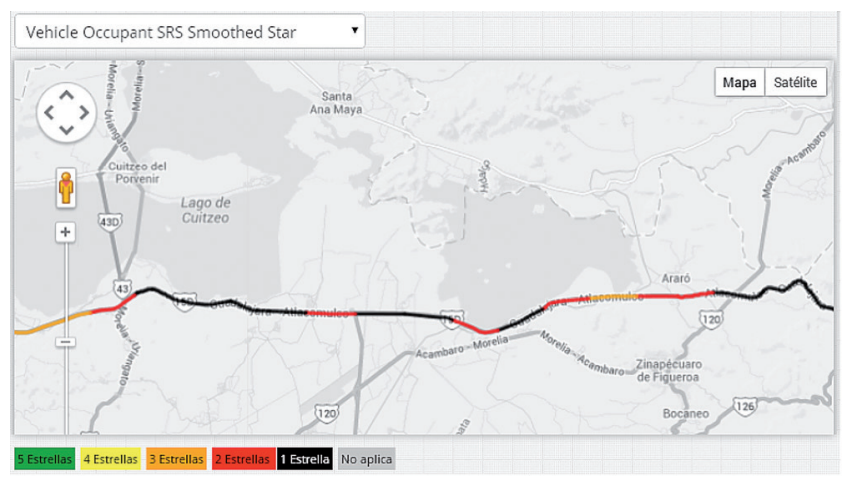

Figura 5. Calificación por estrellas del tramo seleccionado, (cortesía de http://vida.irap.org) condiciones de seguridad mínimas por lo menos deberá contar con una calificación de 3 estrellas.

En la Figura 5 se encuentra la calificación por "estrellas" suavizada (promedio) del tramo seleccionado arrojada por el sistema ViDAiRAP. Como puede observarse, prácticamente el $90 \%$ de su longitud transcurre con una calificación de 1 y 2 estrellas (condiciones de seguridad indeseables) para la condición "Ocupante de Vehículo".

\section{Tablas del sistema ViDA-iRAP}

En la Tabla 2 se muestran las contramedidas y el plan de inversión recomendado por el sistema ViDA-iRAP para toda la longitud de la autopista federal de cuota Maravatío-Zapotlanejo (Mex-15D), Sentido 1.

Entre las principales contramedidas para el tramo seleccionado aparecen la eliminación de peligros laterales (ambos lados), mejoramiento de taludes (ambos lados), colocación de banda alertadora en acotamiento, mejorar señalamiento, y colocación de barrera de orilla (ambos lados).

\section{CONTRAMEDIDAS RECOMENDADAS}

Según el reporte de resultados iRAP 2009 de Chile y Costa Rica, las características típicas que debe tener una carretera, en la condición "Ocupante de Vehículo", para cada una de las categorías de la calificación por "estrellas", son las siguientes:

Tabla 2. Contramedidas con la mayor relación beneficio-costo de la autopista seleccionada (Sentido 1), (cortesía de http://vida.irap.org)

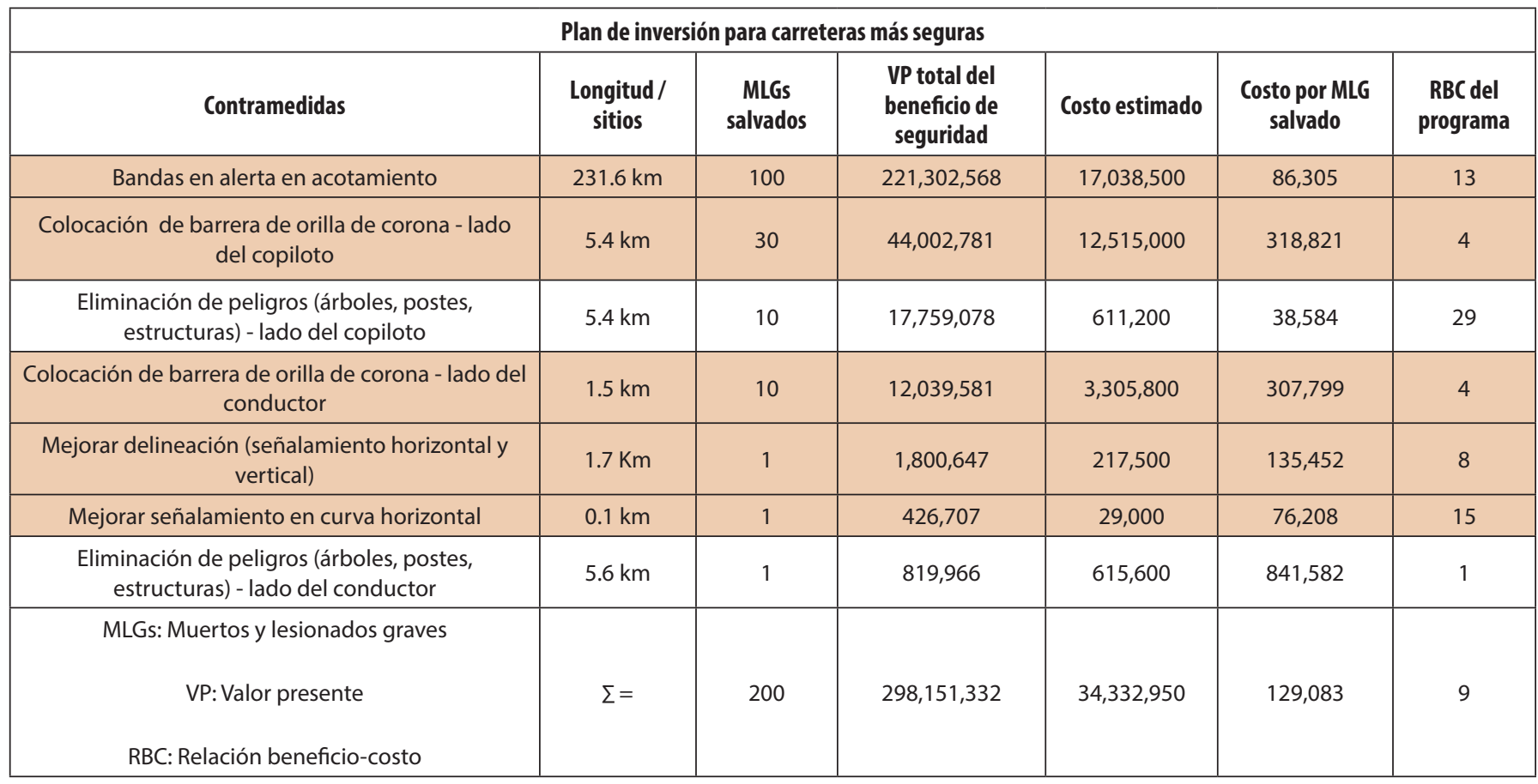


- 5 Estrellas - Cuerpos separados, acotamientos, laterales de la carretera limpios, pocas intersecciones a desnivel con largos carriles de incorporación y límite de velocidad adecuado.

- 4 Estrellas - Sentidos separados con barrera en la mediana, acotamientos, intersecciones a desnivel y buena delineación.

- 3 Estrellas - Carretera de un solo cuerpo, recta, con laterales relativamente libres y acotamientos pavimentados.

- 2 Estrellas - Carretera de un solo cuerpo, con curvas estrechas, acotamientos pavimentados estrechos y obstáculos en los laterales.

- 1 Estrella - Carretera de un solo cuerpo, con fricción lateral (cruces, incorporaciones, etc.) y obstáculos en los laterales.

Con relación a lo anterior, se puede decir que un tramo carretero puede tener una pobre calificación por "estrellas" a pesar de sus características geométricas favorables (cuerpos separados, acotamientos amplios, intersecciones a desnivel, etc.), si a lo largo su trayecto presenta factores de riesgo tales como obstáculos laterales no frangibles, fricciones laterales, taludes inadecuados, etc.; por otro lado, si no se presentaran éstos factores de riesgo las características geométricas que tiene el camino lo limitan a un máximo en su calificación por "estrellas".
En el tramo seleccionado la presencia de obstáculos laterales y de taludes con inclinación inadecuada, resultan ser los elementos que más demeritan la calificación por "estrellas". Ambos problemas pueden resolverse implementando la colocación de barrera de orilla (ambos lados), reforzada con la banda alertadora de acotamiento y el mejoramiento de la delineación (señalamiento), con lo cual, teóricamente, el tramo seleccionado podría aspirar a alcanzar una calificación de 4 a 5 estrellas debido a sus características geométricas. Además, las contramedidas seleccionadas tienen el aspecto a favor de que se encuentran dentro de las actuaciones conocidas de conservación y mantenimiento que la SCT viene aplicando dentro de su red carretera.

En la Tabla 3 se presentan las contramedidas arrojadas por el sistema ViDA-iRAP requeridas en el tramo seleccionado por cada segmento de $100 \mathrm{~m}$ de longitud, aunque el sistema proporciona esta información para todo el tramo estudiado, en la tabla únicamente se muestra, a manera de ejemplo, la información procesada correspondiente a los kilómetros 220 y 239.

Tabla 3. Contramedidas "seleccionadas" requeridas por segmentos de $100 \mathrm{~m}$ para el tramo seleccionado en el km 220 y el km 239 (Sentido 1)

\begin{tabular}{|c|c|c|c|c|c|c|c|c|c|c|}
\hline \multicolumn{11}{|l|}{ Tipo de Contramedida (km 220) } \\
\hline \multicolumn{11}{|l|}{ Mejorar señalamiento en curva horizontal } \\
\hline \multicolumn{11}{|l|}{$\begin{array}{c}\text { Mejorar delineación (señalamiento horizontal } \\
\text { y vertical) }\end{array}$} \\
\hline \multicolumn{11}{|l|}{$\begin{array}{l}\text { Colocación de barrera de orilla - lado } \\
\text { conductor }\end{array}$} \\
\hline \multicolumn{11}{|l|}{ Colocación de barrera de orilla - lado pasajero } \\
\hline \multicolumn{11}{|l|}{ Banda alertadora en acotamiento } \\
\hline Kilometraje Operativo & 220.1 & 220.2 & 220.3 & 220.4 & 220.5 & 220.6 & 220.7 & 220.8 & 220.9 & 221.0 \\
\hline Puntaje SRS (por segmentos de $100 \mathrm{~m}$ ) & 8 & 8 & 15 & 15 & 18 & 8 & 8 & 14 & 42 & 38 \\
\hline Calificación por estrellas suavizada (por tramos) & 2 & 1 & 1 & 1 & 1 & 1 & 1 & 1 & 1 & 1 \\
\hline
\end{tabular}

\begin{tabular}{|c|c|c|c|c|c|c|c|c|c|c|}
\hline \multicolumn{11}{|l|}{ Tipo de Contramedida (km 239) } \\
\hline \multicolumn{11}{|l|}{ Mejorar señalamiento en curva horizontal } \\
\hline \multicolumn{11}{|l|}{$\begin{array}{c}\text { Mejorar delineación (señalamiento horizontal } \\
\text { y vertical) }\end{array}$} \\
\hline \multicolumn{11}{|l|}{$\begin{array}{l}\text { Colocación de barrera de orilla - lado } \\
\text { conductor }\end{array}$} \\
\hline \multicolumn{11}{|l|}{ Colocación de barrera de orilla - lado pasajero } \\
\hline \multicolumn{11}{|l|}{ Banda alertadora en acotamiento } \\
\hline Kilometraje Operativo & 239.1 & 239.2 & 239.3 & 239.4 & 239.5 & 239.6 & 239.7 & 239.8 & 239.9 & 240.0 \\
\hline Puntaje SRS (por segmentos de $100 \mathrm{~m}$ ) & 8 & 42 & 27 & 27 & 34 & 30 & 34 & 34 & 34 & 34 \\
\hline Calificación por estrellas suavizada (por tramos) & 1 & 1 & 1 & 1 & 1 & 1 & 1 & 1 & 1 & 1 \\
\hline
\end{tabular}


La localización de las contramedidas seleccionadas por segmentos de $100 \mathrm{~m}$ de longitud proporcionadas por el sistema ViDA-iRAP, significan un excelente punto de partida para poder cuantificar $y$ presupuestar, con un alto nivel de detalle, el proyecto de mejora de la seguridad operativa de la carretera.

\section{CONCLUSIONES}

- El sistema ViDA-iRAP es una excelente herramienta para agilizar y hacer más eficiente la aplicación de los protocolos que conforman la metodología del programa iRAP, en la detección de los sitios considerados de alto riesgo para los usuarios en materia de seguridad vial; así como, las alternativas de solución para contrarrestar este efecto, y de esta manera coadyuvar con el objetivo del programa iRAP "Carreteras más seguras". Es importante mencionar, que la precisión de la información que el sistema puede proporcionar está supeditada a la calidad de los datos suministrados, ligados a las tareas de inspección y valoración de los atributos de la carretera (codificación). En otras palabras, las etapas de inspección y valoración, representan la base que sostiene a la evaluación de la seguridad vial del camino y consecuentemente a su proyecto de mejora.
- El sistema ViDA-iRAP recomienda contramedidas para cada sección de $100 \mathrm{~m}$ de longitud de la carretera considerada en el estudio. Estas recomendaciones significan un buen punto de partida para realizar los estudios de ingeniería detallados con la finalidad de planificar las mejoras de seguridad más adecuadas para cada sección de la carretera.

- En algunos casos muy particulares ciertas contramedidas propuestas por el sistema ViDA-iRAP podrían tomarse como soluciones redundantes, como la eliminación de obstáculos laterales y la colocación de barreras laterales para un mismo punto del camino. Es necesario que el proyectista emplee su criterio, para seleccionar aquella o aquellas contramedidas que resulten más viables y eficientes en cada punto del camino, tomando en cuenta y sopesando todas las alternativas brindadas por el sistema ViDA-iRAP.

\section{AGRADECIMIENTOS}

Los autores agradecen a la Dirección General de Servicios Técnicos de la Secretaría de Comunicaciones y Transportes, y a la Universidad Michoacana de San Nicolás de Hidalgo, todo el apoyo brindado para la elaboración del presente trabajo.

\section{REFERENCIAS BIBLIOGRAFICAS}

1. Diario Oficial de la Federación (DOF), (6 de junio de 2011). “Acuerdo por el que se da a conocer la Estrategia de Seguridad Vial 2011-2020”. D.F., México.

2. International Road Assessment Programme (iRAP), (2009a). "Resultados iRAP 2009, Chile". Basingstoke Hampshire, UK.

3. International Road Assessment Programme (iRAP), (2009b). “Resultados iRAP 2009, Costa Rica”. Basingstoke Hampshire, UK.

4. International Road Assessment Programme (iRAP), (2012a). “Trustees' report and financial statements for the year ended 31 december 2011”. Basingstoke Hampshire, UK.

5. International Road Assessment Programme (iRAP), (2012b). “Notes from the iRAP innovation workshop, 21-22 June 2012”. TRL, Crowthorne, Berkshire, UK.

6. International Road Assessment Programme (iRAP), (2013a). “The Global Cost of Road Crashes, Fact Sheet”. Basingstoke Hampshire, UK.

7. International Road Assessment Programme (iRAP), (2013b). “iRAP Methodology Fact Sheet \#2, Development History”. Basingstoke Hampshire, UK.

8. International Road Assessment Programme (iRAP), (2013c). “iRAP Methodology Fact Sheet \#7, Star Rating Bands”. Basingstoke Hampshire, UK.

9. International Road Assessment Programme (iRAP), (2013d). “iRAP Methodology Fact Sheet \#6, Star Rating Score Equations”. Basingstoke Hampshire, UK.

10. International Road Assessment Programme (iRAP), (2013e). “iRAP Methodology Fact Sheet \#9, Star Rating Worked Example”. Basingstoke Hampshire, UK.

11. International Road Assessment Programme (iRAP), Web. “News: Rápido progreso en México”. Recuperado de http://www.irap.net/en/new/espanol/319zrapido-progreso-en-mexico.

12. Organización Mundial de la Salud (OMS), (2009). “Informe sobre la situación mundial de la seguridad vial, es hora de pasar a la acción”. Departamento de Prevención de la Violencia y los Traumatismos y Discapacidad. Ginebra, Suiza.

13. Organización Mundial de la Salud (OMS), (2012). “Decenio de Acción para la Seguridad Vial 2011-2020, Lanzamiento Mundial”, Documento WHO/NMH/ VIP12.03. Ginebra, Suiza. 